\title{
Cognitive Mechanisms of Linguistic Manipulation in Advertising Slogans
}

\section{Assel Batyrovna Baidullayeva}

\author{
Abylai khan Kazakh University of International relations and world languages, Almaty, Kazakhstan. Muratbayev str. 200
}

\section{Doi:10.5901/mjss.2015.v6n5s2p270}

\begin{abstract}
The article is concerned with the organization of Russian advertising slogans, which use cognitive mechanisms of linguistic manipulation. It determines the further analysis of communicative advertising strategies (associative and dissociative) and forms the object classification of cognitive manipulation in an advertising slogan, as well as types of connections in an advertising slogan. The latter has laid the basis for the presentation of possible cognitive models of advertising slogans. This article presents the results of empirical study, concerned with searching of the most efficient AS cognitive model from the mentioned above.
\end{abstract}

Keywords: advertising slogan, linguo-semiotic unit, associative strategy, dissociative strategy, implicit linguistic manipulation

\section{Introduction}

Advertisement, represented by advertising texts, is a human activity, which is aimed at solving certain linguistic problems. Its form and content are constantly improving to meet increasing market requirements for the promotion of products and services. An advertising slogan is among the most efficient ways of making a text more market-oriented since it tends to replace the whole advertising text.

An advertising slogan (hereinafter referred to as "AS") presents an independent advertising text or some motto, which conveys a catchy and straightforward message and has an essential connection with its advertising product. Linguists consider an AS to be a linguo-semiotic unit, activating a chain of cognitive and associative bonds to show an advertising object from different perspectives to the best possible advantage (Sandage, Fryburger and Rotzoll, 2004).

From the viewpoint of cognitive linguistics, an AS must be organized in such a way that it reaches the maximum content intensity. According to the general purpose of a text, an AS is defined by an author's objective. It gives the initial impetus to the text creation. Namely, a text is a move towards this objective, determined by an author's will and embodied in a speech act. In terms of communication, the energy level of a speech act (in this case an AS) is directly-proportional to manipulation on a person, for whom this text has been written (addressee), i.e. an author's focused effort (speech addresser) causes comparable manipulation on an addressee (Balabanova, 2003).

Scientists believe that cognitive capacity of an AS comprises the following elements: the rise of the text informative value; the intensification of its emotive features; the activation of implicit linguistic manipulation provided that a linguosemiotic unit preserves its shortest form (Turbina, 2002).

Thus, the rise of the text informative value, the intensification of its emotive features and the activation of implicit linguistic manipulation are basic principles of the AS organization, which determine its form and content. In such a case, the principles mentioned above not only complement each other, but also interact with each other to solve common problems.

The rise of the text informative value is a major feature of any AS. Its short and intense form serves as a primary principle of the AS organization, which is hard to implement, however. An AS draws an addressee's attention to the key statement of an advertisement and presents it in the most laconic way. The implementation of this essential function leads to the intensification of a statement content and the involuntary memorization of connected associations and meanings, caused by an AS. In many cases, accompanying illustrations can increase the AS informative value.

In order to rise the AS informative value, its creator often uses the so-called "precedent texts", which are based upon set phrases, aphorisms, titles of popular songs and books etc.

Modern culture tends to fill new texts with implicit or explicit abstracts and evaluations of earlier texts, as well as rewrite and compile existing texts.

The arising "precedent phenomenon" is built upon social, cultural and linguistic solidarity of an AS creator and an addressee. The amount of borrowed words indicates the value of this source and, as a result, increases its precedent 
characteristics. Each "precedent text" possesses a distinct system of associations, which it awakes in the minds of addressees. Thereby, precedent texts become a unique source of rising the AS informative value (Popova, 2006).

The principle of the informative value rise is closely connected with that of the activation of implicit linguistic manipulation. The last one is used when reporting information is not verbally expressed (remains non-verbalized) and is conveyed by covert linguistic elements. In other words, this information does not get an explicit form, however it is referred by an AS.

The vast majority of statements contains implicit information, which is merely implied. This information embraces the whole complex of cognitive mechanisms of linguistic manipulation therefore the use of implicit information becomes significant for the creation of an AS. As opposed to explicit information, contained in an AS, implicit information is not usually comprehended by an addressee. It influences a recipient bypassing analytical operations for the processing of received information. In this regard, an addressee does not evaluate, criticize or doubt implicit information. While mentally processing implicit information, an addressee uses everyday knowledge, including the knowledge of lifestyles, social and cultural stereotypes and the characteristics of an applied language or other semiotic systems.

In case when an AS message is expressed directly, i.e. explicitly, it can be regarded by a recipient as a way of imposing another person's opinion and consequently be rejected. While the message, expressed implicitly, is not directly evaluated and is interpreted by an addressee as an individual conclusion. It makes an AS much more efficient (Pirogova, 2001).

The principle of the activation of implicit linguistic manipulation is also connected with the principle of the intensification of AS emotive features since the creator of an AS pursues an objective of intensifying the emotive component of a communicative act, which leads an addressee to make rather an emotional than a rational decision. Herein the expression of an AS should correspond with a posed communicative task, which is to extract all abundant elements from an AS.

This universal characteristic of the speech unit actualization is especially profound in an AS because the AS form aims at the utmost laconism and the highest expressiveness. It is the implicit information of an AS that intensifies its emotive features. The more extensive and more associative this information is, the higher the AS emotive level is.

The listed above principles of the rise of the text informative value, the intensification of its emotive features and the activation of implicit linguistic manipulation are mainly implemented through the AS syntactic structure.

Various syntactic pauses, parceling sentences and elliptical constructions enable to use the contained implicit information in the most efficient and diverse way. As a result, syntactic implication is formed, which is based upon the awareness that "something should be (and exists somewhere) but is not directly expressed" (Popova \& Sternin, 2006).

Thus, the L'Oréal company, a cosmetics producer, conveys the following message, "As you are the best, you deserve the cosmetic product, which is \#1 on the market, and it is the very product we offer you because it is the best". Let's examine the original AS of L'Oréal -"Because you're worth it". The AS presents a subordinate clause (causal). However, a principal clause, which expresses cause-and-effect relations, is absent. This principal part of the sentence should be considered by each addressee. Moreover, a recipient should decide why the advertising product is meant for him or her in particular.

The principles of the AS organization being described, we should analyze basic communicative strategies of advertisement, which adopt cognitive mechanisms of linguistic manipulation, and study the classification of objects for cognitive manipulation and types of relations in an AS.

In scientists' opinion, the most significant strategic task for a copywriter is comparative positioning (Trout, 2001). It comprises the creation of the unique image of an advertising object (the main objectives are to stress out its competitive advantages, to capture a certain market segment etc.) and its connection with positive associations of addressees.

Therefore, an AS aims at creating the image of an advertising object. Following scientists, we would regard it as a solid representation of various characteristics of this advertising object, both rational and emotional. The image of an advertising object being created, advertisement often strives to connect an advertising object with addressee's positive associations.

However, scientists think modern advertisement is also characterized by the opposition of cognitive meanings. In this respect, advertisement not only emphasizes the positive characteristics of an advertising object but also allays addressee's concerns to convince a person that buying a product or a service will not bring any problems (Goryachev, 2009). Furthermore, advertisement frequently exploits human fears, giving positive and negative motivations.

Hence, two communicative advertising strategies can be distinguished: the associative strategy, which stresses out connections between different objects, and the dissociative strategy, which lays an emphasis on distinct features of an advertising object and its separation from the mental image of other objects, phenomena or ideas. The associative strategy forms positive marks of a grading scale and the dissociative strategy organizes its negative marks. 
For instance, the AS of ASTON MARTIN "Power. Beauty. Soul" shows certain characteristics as positive ones while the AS of LAND CRUISER Prado "The car, which is not given to others" presents the advertising object by stating that it is different from "others".

While analyzing the mechanisms of the associative and dissociative strategies in an AS, it would be useful to examine, firstly, elements of advertising connections and, secondly, types of these connections.

There are various elements, expressing advertising connections in an AS, however scientists point out "basic" ones, which are typical of the advertising discourse. Namely, they are an advertising object, an advertising addressee and advantages an addressee gets from buying this AS object.

While analyzing an AS, one should define how elements of advertising connections are expressed in this AS and what types of connections are formed between them in the process of the AS comprehension.

The analysis of an advertising object has shown that an AS uses different techniques to immerse an advertising object into the text. Nevertheless, the choice of a certain technique greatly depends on the AS syntactic structure. All ASs can be divided into two groups in accordance with their syntactic structure. The first group contains ASs, which represent a part of a bigger construction where a trademark reference defines an advertising object, for instance, "How nice it is to have a Farm House". The second group comprises ASs, which are made of separate sentences, for example, "Herschi Cola. The taste of victory!".

In the former case an advertising object in an AS is expressed indirectly and implicitly, while in the latter case it is represented by a hyperonym ("Patra. Beer with a plug") or by a different part of speech ("Gillette. The best a man can get").

The level of explicitness in an AS advertising object can vary from the utmost self-determination ("OMSA tights are known for being good from Paris to Surgut!") to the total absence of an advertising object in an AS, which causes ambiguity on pragmatic level ("Russian Radio. Everything is gonna be alright").

The next element of advertising connections is an addressee. In general, an addressee is the collective representation of a "target group" (TG), which has similar consumer preferences and traditionally belongs to one social class. If an advertising addressee is directly indicated in an AS, this person acts like a typical representative of the TG or the so-called "opinion leader", i.e. a representative of a social class referential for a consumer (llin, 2000). As a rule, this advertising text is presented in a form of direct speech. It is mostly typical of TV advertisement but can be also used in an AS, for example, "Do you doubt my good taste?" (Winston).

However, there are some linguistic features, which subtly shape the generalized character of an advertising addressee. In this case, scientists believe the usage of personal pronouns and imperative sentences (direct or indirect) is quite symptomatic.

Then an advertising addressee should get an overview of the potential advantages obtained after advertising. Some ASs specify addressee's advantages by using special nominations, for example, "Clear Vita Abe. No dandruff just soft hair" or "Beeline. Being with us is convenient". Others make an addressee searching for this advantage. This indirect representation of potential advantages can be seen in the following AS - SUPRADIN. "Does your life stop with the end of a workday?".

After advertising objects and addressees in an AS have been thoroughly surveyed, we should analyze the way advertising object and its addressee correlate with mental representations of other objects, as well as define the nature of these objects.

Cognitive linguistics widely uses the term "connective", i.e. the method of connection. By describing connectives, we can characterize various relations between elements of mental space (Guillaume, 1992).

\section{Techniques}

The analysis of various connections between AS elements enables us to characterize cognitive mechanisms of linguistic manipulation in an AS. We can distinguish the following types of connections of mental representations ( $A$ is the representation of an advertising object and $B$ is the representation of another object):

1. Identity, i.e. $A$ is equal to $B(A=B)$. For instance, in the AS "The tastiest remedy against caries" (chewing gum Orbit) the consumption of the advertising object (namely, the brand of chewing gums) is made equal to a medicine against caries. The same technique is used in the AS "A taste of paradise" (chocolate bar Bounty).

2. Inclusion, i.e. an advertising object is included into some category (A refers to category B). For example, the category of the AS "Das Auto!" (VOLKSWAGEN) is quite obvious since the car is included into the exclamatory category. The AS "Even milk is tastier in Milky Way!" puts the advertising object into the cognitive category "dairy products", i.e. Milky Way is shown not only as a chocolate bar (sweets) but also as a dairy 
product (wholesome).

3. Intercrossing (A and B have something in common). For instance, "It's Beaujolais Nouveau Time!" (wine Beaujolais Nouveau). The brand name is connected with the event name.

4. Transformation (A influences and changes B). For example, "Philips. Let's make things better", "Indesit. We work, you play".

5. Designation (A is designated for B), i.e. it is implied that B has A. For instance, the AS "Mazda. If you dare" connects its advertising object with a familiar address, which is directed at everyone who dares, "Mesim. Your stomach cannot go without it!", "Temptation for the most sophisticated" (beer Holsten).

6. Superiority (the quality of $A$ is either more or less than that of $B$ ). For example, "Vanish. More than a stain remover", "More than television" (satellite television NTV).

7. Opposition (A is not like B or A is different from B). For instance, "Some things age, others mature" (whisky Dewar's 12 Special Reserve), "Women were not born for washing!" (washing machines Hansa, Germany), designed for the certain TG, i.e. women, "Princess. Just like your mother's, only better!" (girls cosmetics Princess), "Strong Enough for a Man, Made for a Woman" (female deodorant Secret).

Depending on the reference of an advertising objects and the type of its connection, the following commonly used AS cognitive models can be mentioned:

1. Advertising object $\rightarrow$ (transformation) $\rightarrow$ addressee.

For example, the AS "Power your brain with Nuts" shows the influence of the advertising object (chocolate bar Nuts) on an addressee. The AS "Golden Lady. Grande Amica" (tights Golden Lady) mentions the influence of the advertising object on a reference and numerous group (women), which is so large that its identity to an addressee is merely implied.

2. Advertising object $\rightarrow$ (superiority) $\rightarrow$ alternative advertising object.

For instance, the AS "Tele-2. Always cheaper" and "More than television" (satellite television NTV) postulate the superiority of the advertising object over some alternative object, which is not directly named (since the express reference to business rivals is restricted by law).

3. Advertising object $\rightarrow$ (designation) $\rightarrow$ addressee.

For example, "For those, who are on the way" (Autoradio), "For Life" (car brand "Volvo") and "For those, who like their teeth" (PRESIDENT, toothpaste).

\section{Results}

The empirical study has been conducted in order to choose the most efficient AS cognitive model among the above mentioned.

The study objects are the ASs of women underwear "Golden Lady. Grande Amica" (model 1: advertising object $\rightarrow$ (transformation) $\rightarrow$ addressee), abstergents "Vanish. More than a stain remover" (model 2: advertising object $\rightarrow$ (superiority) $\rightarrow$ alternative advertising object) and hygiene products "For those, who like their teeth" (PRESIDENT, toothpaste) (model 3: advertising object $\rightarrow$ (designation) $\rightarrow$ addressee).

The survey sample includes two groups of forty people each. The first group consists of unmarried young women from 20 to 25 years old. The second group is made of married women from 30 to 35 years old.

The survey was being conducted within two months in two stages:

1. Interaction with the female audience of 20-25 years old for the evaluation of AS cognitive manipulation, using a grading scale system and the in-hall test.

2. Interaction with the female audience of 30-35 year old for the evaluation of AS cognitive manipulation, using the same techniques.

The AS evaluation, using the in-hall test, was being carried out on case-by-case basis with two respondents a day within two weeks. The procedure of the in-hall test is described below. The tested material was recorded on a DVD and then showed on a computer monitor. The AS evaluation of the abstergent, using the in-hall test, was carried out in the similar way.

The grading evaluation of AS cognitive manipulation was conducted within a group one week after the in-hall test. Each AS evaluation took one day.

Here we present a brief summary of the used techniques.

The in-hall method is based upon the Impact and Communication Test. Its essence is as follows. Firstly, an addressee of advertising manipulation watches a TV-program with a commercial break for 20 minutes. The commercial break includes three tested advertising clips. Thus, examinees are not aware of an AS being tested. They are 
intentionally given an impression that it is the TV-program under the test. In the end of the viewing examinees answer some questions, which are recorded and then analyzed. Since commercial clips with various AS cognitive models were shown, it gave us an opportunity to compare the efficiency of these models.

The first question pool of the in-hall test allowed us to measure the brand and advertising image recall, to verify the AS understanding, to estimate it on the scale "like/dislike" and to find out an attitude towards an advertising object. After receiving the answers to the first question pool, the tested advertising clips were shown repeatedly without an accompanying TV-program. As examinees watched these commercials for the second time, they paid more attention to them, it allowed us to evaluate qualitative AS characteristics, namely, the understanding of AS meaning, the understanding of an advertising object, the advantage of the advertising object acquisition, the attitude towards an advertising object and its AS. The analysis of the in-hall test results was based on the following parameters: Top of Mind (the number of respondents (\%), who were the first to recall the presented AS; the AS recall rate; average score of originality (a five-grade scale).

With the help of a five-grade scale, measuring AS cognitive manipulation, the AS perception and the easiness of its content memorizing are analyzed. The five-grade scale of AS cognitive manipulation has the following structure:

- grade 1: an addressee tries to avoid AS manipulation by turning off the TV-set and so on. At the same time an addressee has strong negative emotions. Moreover, it does not matter whether an addressee recalls the AS content and an advertising object itself or not. An addressee does not intend to buy an advertising object;

- grade 2: an addressee is indifferent to an AS. It does not awake any feelings. A person does not memorize the AS content or an advertising object itself. An addressee does not intend to buy an advertising object;

- grade 3: an AS attracts addressee's attention, sparks interest and curiosity. An addressee recalls the AS content without correlating it with an advertising object. An addressee still does not want to buy an advertising object. An AS seems to exist independently, detached from an advertising object. In an addressee's consciousness an advertising object and an AS are not associated with each other;

- grade 4: an AS attracts an addressee's attention, sparks interest and curiosity. An addressee recalls the AS content as well as an advertising object. An addressee seemingly agrees to buy an advertising object. Although a person is not ready to do it immediately, an addressee thinks about it;

- grade 5: an AS attracts an addressee's full attention and sparks a lot of interest. An addressee has strong positive emotions. An addressee recalls both the AS content and an advertising object. An addressee has a strong desire to buy an advertising object.

\section{Discussion}

The in-hall test has given the following results.

In the first female age group (20-25 years) the quantitative characteristics (\% of the respondents number) of AS qualitative characteristics are distributed as follows:

model 1 (underwear AS "Golden Lady. Grande Amica": advertising object $\rightarrow$ (transformation) $\rightarrow$ addressee): Top of Mind (45\%); the number of respondents, recall rate (70\%); average score of originality -4.5 .

model 2 (abstergent AS "Vanish. More than a stain remover": advertising object $\rightarrow$ (superiority) $\rightarrow$ alternative advertising object): Top of Mind (25\%); recall rate (50\%); average score of originality -3.5 .

model 3 (hygiene product AS "For those, who like their teeth" (PRESIDENT, toothpaste): advertising object $\rightarrow$ (designation) $\rightarrow$ addressee): Top of Mind (30\%); recall rate (60\%); average score of originality -4.0 .

In the second female age group (30-35 years) the quantitative characteristics ( $\%$ of the number of respondents) of AS qualitative characteristics are distributed as follows:

model 1 (underwear AS "Golden Lady. Grande Amica": advertising object $\rightarrow$ (transformation) $\rightarrow$ addressee): Top of Mind (50\%); the number of respondents, recall rate (75\%); average score of originality -4.0 .

model 2 (abstergent AS "Vanish. More than a stain remover": advertising object $\rightarrow$ (superiority) $\rightarrow$ alternative advertising object): Top of Mind (30\%); recall rate (45\%); average score of originality- 3.5 .

model 3 (hygiene product AS "For those, who like their teeth" (PRESIDENT, toothpaste): advertising object $\rightarrow$ (designation) $\rightarrow$ addressee): Top of Mind (20\%); recall rate (40\%); average score of originality -3.5 .

The correlation of the in-hall test results has shown that in both female age groups the most efficient is the cognitive model 1, its AS is based upon the transformative manipulation of the advertising object and its impact on an addressee.

The grading results of advertising cognitive manipulation have confirmed the in-hall test results: model 1 - average score 4.2 in the first age group and 4.3 - in the second age group; 
model 2 - average score 3.4 in the first age group and 3.5 - in the second age group; model 3 - average score 3.6 in the first age group and 3.8 - in the second age group.

In this manner, it can be concluded that among the three models of the cognitive manipulation the most preferable one is model 1: advertising object $\rightarrow$ (transformation) $\rightarrow$ addressee.

\section{Conclusion}

In the conclusion it should be noted that, the rise of the text informative value, the intensification of its emotive features and the activation of implicit linguistic manipulation represent the basic principles of the AS organization. They form the AS intensity, determining the degree of influence on an addressee. At the same time the AS informative value is a fundamental indicator. The AS emotive features and its informative value are interdependent, as they do not oppose each other but rather interact. The analysis of possible types of connections between AS elements allows us to switch to the AS cognitive models representation, which gives us a chance to characterize cognitive mechanisms of AS manipulation in greater details. In the conclusion, it should be mentioned that the presented analysis scheme, based upon the facts of modern cognitive linguistics, can be used to identify the reasons of AS success and to create the most efficient cognitive AS.

\section{References}

Sandage, C., V. Fryburger and K. Rotzoll, 2004. Advertising: Theory and Practice. Moscow: SMART.

Balabanova, I. Y., 2003. Axiology of the Advertising Text. Russian Comparative Philology: Systemic-Functional Aspect. Kazan, pp: 1823.

Turbina, O.A., 2002. The Principles of the Linguistic Implicitness Study. Linguistic Subjects and Language Teaching Methods in the HEI. Chelyabinsk: SUSU.

Popova, E.S., 2006. Prototype Advertising Text in the Structure of Linguistic Manipulation. The Word. The Dictionary. Philology: Sociocultural Coordinates. Saint-Petersburg, pp: 103-108.

Pirogova, U.K., 2001. The Implicit Information as an Instrument of the Linguistic Impact and Manipulation (based on advertising materials). The Problems of Applied Linguistics. Moscow.

Popova, Z.D. and I.A. Sternin, 2006. Cognitive-Semantic Analysis of the Language. Voronezh: Origins, pp: 26.

Trout, D., 2001. New Positioning. Saint-Petersburg.

Goryachev, A.A., 2009. The Strategies of Advertising Image Creation. Communicative Studies 2009: Communication Types. Learning to Communicate: Continuing Scientific Publication. Sc. edit I. A. Sterning. Voronezh: Origins, pp: 28-41.

Ilin, V.I., 2000. Consumers' Behavior. Saint-Petersburg.

Guillaume, G., 1992. Principles of the Theoretical Linguistics. Moscow: Progress. 